\title{
Production and sequential decay of charmed hyperons
}

\author{
Göran Fäldt ${ }^{*}$ \\ Department of Physics and Astronomy, Uppsala University, Box 516, S-751 20 Uppsala, Sweden
}

(Received 21 December 2017; published 12 March 2018)

\begin{abstract}
We investigate production and decay of the $\Lambda_{c}^{+}$hyperon. The production considered is through the $e^{+} e^{-}$ annihilation channel, $e^{+} e^{-} \rightarrow \Lambda_{c}^{+} \bar{\Lambda}_{c}^{-}$, with summation over the $\bar{\Lambda}_{c}^{-}$antihyperon spin directions. It is in this situation that the $\Lambda_{c}^{+}$decay chain is identified. Two kinds of sequential decays are studied. The first one is the doubly weak decay $B_{1} \rightarrow B_{2} M_{2}$, followed by $B_{2} \rightarrow B_{3} M_{3}$. The other one is the mixed weakelectromagnetic decay $B_{1} \rightarrow B_{2} M_{2}$, followed by $B_{2} \rightarrow B_{3} \gamma$. In both schemes $B$ denotes baryons and $M$ mesons. We should also mention that the initial state of the $\Lambda_{c}^{+}$hyperon is polarized.
\end{abstract}

DOI: 10.1103/PhysRevD.97.053002

\section{INTRODUCTION}

We shall investigate properties of certain sequential decays of the $\Lambda_{c}^{+}$hyperon, but in order to do so we first need to produce them. To this end we consider the reaction $e^{+} e^{-} \rightarrow \Lambda_{c}^{+} \bar{\Lambda}_{c}^{-}$, which is analyzed in detail in Refs. [1,2]. In order to describe such an annihilation process two hadronic form factors are needed, commonly denoted $G_{E}$ and $G_{M}$. They can be parametrized by two parameters, $\alpha$ and $\Delta \Phi$, with $-1 \leq \alpha \leq 1$. For their precise definitions we refer to Ref. [2]. At the $\Lambda_{c} \bar{\Lambda}_{c}$ threshold $G_{E}=G_{M}$ leading to a vanishing $\Delta \Phi$.

The general cross-section distribution of this annihilation reaction depends on six structure functions which themselves are functions of $\alpha, \Delta \Phi$, and $\theta$, the scattering angle. In our application, however, we sum over the decay products of the antihyperon $\bar{\Lambda}_{c}^{-}$, but identify the decay chain of the hyperon $\Lambda_{c}^{+}$, so called single tag events. In this simplified case only two structure functions are relevant,

$$
\begin{gathered}
\mathcal{R}=1+\alpha \cos ^{2} \theta, \\
\mathcal{S}=\sqrt{1-\alpha^{2}} \sin \theta \cos \theta \sin (\Delta \Phi) .
\end{gathered}
$$

The scattering distribution function for the $\Lambda_{c}^{+}$ hyperon production becomes, according to Refs. [1,2], proportional to

$$
W(\mathbf{n})=\mathcal{R}+\mathcal{S} \mathbf{N} \cdot \mathbf{n},
$$

\footnotetext{
*goran.faldt@physics.uu.se
}

Published by the American Physical Society under the terms of the Creative Commons Attribution 4.0 International license. Further distribution of this work must maintain attribution to the author(s) and the published article's title, journal citation, and DOI. Funded by SCOAP ${ }^{3}$. where $\mathbf{n}$ is the direction of the hyperon spin vector in the hyperon rest system, $\mathbf{N}$ the normal to the scattering plane,

$$
\mathbf{N}=\frac{1}{\sin \theta} \hat{\mathbf{p}} \times \hat{\mathbf{k}}
$$

and $\cos \theta=\hat{\mathbf{p}} \cdot \hat{\mathbf{k}}$. The momenta $\mathbf{k}$ and $\mathbf{p}$ are the relative momenta in the initial and final states in the c.m. (center of momentum) system, i.e.,

$$
\begin{aligned}
& \mathbf{k}=\mathbf{k}_{1}=-\mathbf{k}_{2} \\
& \mathbf{p}=\mathbf{p}_{1}=-\mathbf{p}_{2} .
\end{aligned}
$$

The spin four-vector $s$ of a particle of four-momentum $p$ satisfies $s \cdot p=0$ [3], and in the particle rest system it simplifies to $s=(0, \mathbf{n})$, with the three-vector $\mathbf{n}$ a unit vector, $\mathbf{n} \cdot \mathbf{n}=1$.

From Eq. (1.3) we deduce for the spin-density distribution function,

$$
S(\mathbf{P})=1+\mathbf{P} \cdot \mathbf{n}
$$

and $\mathbf{P}$ the hyperon polarization,

$$
\mathbf{P}=(\mathcal{S} / \mathcal{R}) \mathbf{N},
$$

subject to the restriction $|\mathbf{P}| \leq 1$. At threshold, $\Delta \Phi$ vanishes and so does $\mathcal{S}$ and the polarization $\mathbf{P}$. For an unpolarized initial-state hyperon $\mathbf{P}=0$.

\section{WEAK HYPERON DECAYS}

The weak hyperon decay $c \rightarrow d \pi$, of which $\Lambda \rightarrow p \pi^{-}$is an example, is described by two amplitudes, one S-wave and one P-wave amplitude. The spin-density distribution function of the decay is commonly characterized by three 
parameters, denoted $\alpha \beta \gamma$. They are not independent but fulfill the relation

$$
\alpha^{2}+\beta^{2}+\gamma^{2}=1 .
$$

This parametrization is discussed in detail in Ref. [4] and also in Ref. [1].

We denote by $G_{c}(c, d)$ the joint spin-density distribution function for the weak hyperon decay $c \rightarrow d \pi$, given the spin vectors $\mathbf{n}_{c}$ and $\mathbf{n}_{d}$,

$G_{c}(c, d)=1+\alpha_{c} \mathbf{n}_{c} \cdot \mathbf{l}_{d}+\alpha_{c} \mathbf{n}_{d} \cdot \mathbf{l}_{d}+\mathbf{n}_{c} \cdot \mathbf{L}_{c}\left(\mathbf{n}_{d}, \mathbf{l}_{d}\right)$,

with

$$
\mathbf{L}_{c}\left(\mathbf{n}_{d}, \mathbf{l}_{d}\right)=\gamma_{c} \mathbf{n}_{d}+\left[\left(1-\gamma_{c}\right) \mathbf{n}_{d} \cdot \mathbf{l}_{d}\right] \mathbf{l}_{d}+\beta_{c} \mathbf{n}_{d} \times \mathbf{l}_{d} .
$$

The vector $\mathbf{l}_{d}$ is a unit vector in the direction of motion of the decay baryon $d$ in the rest system of baryon $c$. The indices on the $\alpha \beta \gamma$ parameters remind us they characterize hyperon $c$. We repeat that $\mathbf{n}_{c}$ and $\mathbf{n}_{d}$ are the directions of the spin vectors of baryons $c$ and $d$ in their respective rest systems.

Since the spin of baryon $d$ is often not measured, the relevant spin-density distribution function of hyperon $c$ is obtained by averaging over the spin directions $\mathbf{n}_{d}$,

$$
\begin{aligned}
W_{c}\left(\mathbf{n}_{c} ; \mathbf{l}_{d}\right) & =\left\langle G_{c}(c, d)\right\rangle_{d} \\
& =U_{c}+\mathbf{n}_{c} \cdot \mathbf{V}_{c},
\end{aligned}
$$

with

$$
U_{c}=1, \quad \mathbf{V}_{c}=\alpha_{c} \mathbf{l}_{d} .
$$

The notation $\langle--\rangle_{d}$ is a shorthand notation for average over the directions of the spin vector $\mathbf{n}_{d}$, following the prescription of Ref. [1],

$$
\langle 1\rangle_{\mathbf{n}}=1, \quad\langle\mathbf{n}\rangle_{\mathbf{n}}=0, \quad\langle\mathbf{n} \cdot \mathbf{k n} \cdot \mathbf{l}\rangle_{\mathbf{n}}=\mathbf{k} \cdot \mathbf{l} .
$$

Hyperons we study are produced in some reaction, and their states are described by some spin-density distribution function, Eq. (1.7),

$$
S_{c}\left(\mathbf{P}_{c}\right)=1+\mathbf{P}_{c} \cdot \mathbf{n}_{c} .
$$

The spin-density distribution function for the production of a hyperon followed by its decay is obtained by a contraction of the products of the spin-densities for the production and decay steps. The contraction involves averages over initial and final hyperon spin directions $\mathbf{n}_{c}$ and $\mathbf{n}_{d}$,

$$
\begin{aligned}
W_{c}\left(\mathbf{P}_{c} ; \mathbf{l}_{d}\right) & =\left\langle S_{c}\left(\mathbf{P}_{c}\right) G_{c}(c, d)\right\rangle_{c d} \\
& =1+\mathbf{P}_{c} \cdot \mathbf{V}_{c}
\end{aligned}
$$

where $\mathbf{V}_{c}=\alpha_{c} \mathbf{l}_{d}$, from Eq. (2.5). The $c d$ double index in Eq. (2.8) indicates averages over both $\mathbf{n}_{c}$ and $\mathbf{n}_{d}$ according to the prescription (2.6).

From Eq. (2.8) it is clear that if the polarization is known the asymmetry parameter $\alpha_{c}$ can be measured, but not the $\beta_{c}$ or $\gamma_{c}$ parameters. For that to be possible we must measure the polarization of the decay baryon $d$. If hyperon $c$ is produced within a $c \bar{c}$ pair in $e^{+} e^{-}$annihilation then its polarization can be determined from the cross-section distribution.

\section{ELECTROMAGNETIC HYPERON TRANSITIONS}

Electromagnetic transitions such as $\Sigma^{0} \rightarrow \Lambda \gamma$ and $\Xi^{0} \rightarrow \Lambda \gamma$ can also be studied in $\Lambda_{c}^{+}$decays.

An electromagnetic transition $c \rightarrow d \gamma$ is described by a spin-density distribution function similar to that of the weak decay, Eq. (2.2). However, the special feature of the electromagnetic interaction is the photon helicity which can take only two values, $\lambda_{\gamma}= \pm 1$.

The electromagnetic transition distribution function corresponding to Eq. (2.2) is

$G_{\gamma}\left(c d ; \lambda_{\gamma}\right)=\left(1-\mathbf{n}_{c} \cdot \mathbf{l}_{d} \mathbf{l}_{d} \cdot \mathbf{n}_{d}\right)-\lambda_{\gamma}\left(\mathbf{n}_{c} \cdot \mathbf{l}_{d}-\mathbf{n}_{d} \cdot \mathbf{l}_{d}\right)$,

where $\mathbf{l}_{d}$ is a unit vector in the direction of motion of hyperon $d$ in the rest system of hyperon $c$.

Averaging over photon polarizations the transition distribution takes a simpler form,

$$
G_{\gamma}(c, d)=1-\mathbf{n}_{c} \cdot \mathbf{l}_{d} \mathbf{l}_{d} \cdot \mathbf{n}_{d} .
$$

We notice that when both hadron spins are parallel or antiparallel to the photon momentum, then the transition probability vanishes, a property of angular-momentum conservation. We also notice that expression (3.2) cannot be written in the $\alpha \beta \gamma$ representation of Eq. (2.2).

\section{TWO-STEP WEAK HYPERON DECAY}

Now, we apply the above technique to hyperons decaying in two steps, such as $b \rightarrow c \rightarrow d$, accompanied by pions. An example of this decay mode is $\Lambda_{c}^{+} \rightarrow \Lambda \pi^{+}$ followed by $\Lambda \rightarrow p \pi^{-}$.

We denote by $G_{b}(b, c)$ the spin-density distribution function describing the hyperon decay $b \rightarrow c \pi$ pertaining to spin vectors $\mathbf{n}_{b}$ and $\mathbf{n}_{c}$,

$G_{b}(b, c)=1+\alpha_{b} \mathbf{n}_{b} \cdot \mathbf{l}_{c}+\alpha_{b} \mathbf{n}_{c} \cdot \mathbf{l}_{c}+\mathbf{n}_{b} \cdot \mathbf{L}_{b}\left(\mathbf{n}_{c}, \mathbf{l}_{c}\right)$, 
with

$$
\mathbf{L}_{b}\left(\mathbf{n}_{c}, \mathbf{l}_{c}\right)=\gamma_{b} \mathbf{n}_{c}+\left[\left(1-\gamma_{b}\right) \mathbf{n}_{c} \cdot \mathbf{l}_{c}\right] \mathbf{l}_{c}+\beta_{b} \mathbf{n}_{c} \times \mathbf{l}_{c} .
$$

The vector $\mathbf{l}_{c}$ is a unit vector in the direction of motion of baryon $c$ in the rest system of baryon $b$.

By folding the two spin-density distribution functions $G_{b}(b, c)$ and $G_{c}(c, d)$, i.e., averaging their product over the spin vectors $\mathbf{n}_{c}$ and $\mathbf{n}_{d}$ according to prescription (2.6), we get the decay-density distribution function

$$
\begin{aligned}
W_{b}\left(\mathbf{n}_{b} ; \mathbf{l}_{c}, \mathbf{l}_{d}\right) & =\left\langle G_{b}(b, c) G_{c}(c, d)\right\rangle_{c d} \\
& =U_{b}+\mathbf{n}_{b} \cdot \mathbf{V}_{b},
\end{aligned}
$$

with

$$
\begin{gathered}
U_{b}=1+\alpha_{b} \alpha_{c} \mathbf{l}_{c} \cdot \mathbf{l}_{d}, \\
\mathbf{V}_{b}=\alpha_{b} \mathbf{l}_{c}+\alpha_{c} \mathbf{L}_{b}\left(\mathbf{l}_{d}, \mathbf{l}_{c}\right) .
\end{gathered}
$$

The result is interesting. In many cases the asymmetry parameter $\alpha_{c}$ for the $c$ hyperon and the polarization $\mathbf{P}_{b}$ for the initial-state $b$ hyperon are known. Then, just as in the single-step case of Eq. (2.7), the initial state is described by a spin-density distribution function

$$
S_{b}\left(\mathbf{P}_{b}\right)=1+\mathbf{P}_{b} \cdot \mathbf{n}_{b}
$$

For the decay distribution of a polarized hyperon, we obtain

$$
\begin{aligned}
W_{b}\left(\mathbf{P}_{b} ; \mathbf{l}_{c}, \mathbf{l}_{d}\right) & =\left\langle S_{b}\left(\mathbf{P}_{b}\right) G_{b}(b, c) G_{c}(c, d)\right\rangle_{b c d} \\
& =U_{b}+\mathbf{P}_{b} \cdot \mathbf{V}_{b} .
\end{aligned}
$$

This is equivalent to making the replacement $\mathbf{n}_{b} \rightarrow \mathbf{P}_{b}$ in Eq. (4.3).

We conclude that by determining $U_{b}$ and $\mathbf{V}_{b}$ of Eqs. (4.4) and (4.5), we should be able to determine all three decay parameters $\alpha_{b}, \beta_{b}$, and $\gamma_{b}$, for the $b$ hyperon, and $\alpha_{c}$ for the $c$ hyperon.

It is now clear how to get the cross-section distribution for production of $\Lambda_{c}^{+}$in $e^{+} e^{-}$annihilation and its subsequent decay $\Lambda_{c}^{+} \rightarrow \Lambda \pi^{+}$and $\Lambda \rightarrow p \pi^{-}$. Starting from the expressions for the scattering distribution function, Eq. (1.3), and the polarization, Eq. (1.8), we obtain

$$
\mathrm{d} \sigma \propto\left[\mathcal{R} U_{\Lambda_{c}}+\mathcal{S} \mathbf{N} \cdot \mathbf{V}_{\Lambda_{c}}\right] \mathrm{d} \Omega_{\Lambda_{c}} \mathrm{~d} \Omega_{\Lambda} \mathrm{d} \Omega_{p},
$$

with $\mathbf{N}$, Eq. (1.4), the normal to the scattering plane. The functions $\mathcal{R}$ and $\mathcal{S}$ are defined in Eqs. (1.1) and (1.2) and depend among other things on the $\Lambda_{c}^{+}$scattering angle $\theta$ $\left(=\theta_{\Lambda_{c}}\right)$. In Eqs. (4.4) and (4.5) indices are interpreted as; $b=\Lambda_{c}^{+}, c=\Lambda, d=p$.
When integrating over the decay angles $\Omega_{\Lambda}$ and $\Omega_{p}$ in Eq. (4.8) we observe that the term involving the polarization $\mathbf{N} \cdot \mathbf{V}_{\Lambda_{c}}$ vanishes, as does the term involving the angular dependent part of $U_{\Lambda_{c}}$. This results is the crosssection distribution of Eq. (1.1),

$$
\mathrm{d} \sigma \propto\left[1+\alpha \cos ^{2} \theta_{\Lambda_{c}}\right] \mathrm{d} \Omega_{\Lambda_{c}},
$$

describing the annihilation reaction $e^{+} e^{-} \rightarrow \Lambda_{c}^{+} \bar{\Lambda}_{c}^{-}$.

It is more interesting to perform a partial integration. Let us integrate over the angles $\Omega_{\Lambda}$ and $\Omega_{p}$ keeping $\cos \theta_{\Lambda p}$ of

$$
\cos \theta_{\Lambda p}=\mathbf{l}_{\Lambda} \cdot \mathbf{l}_{p}
$$

constant. Also in this case does the contribution involving the polarization vanish. We are left with

$$
\begin{aligned}
\mathrm{d} \sigma \propto & {\left[1+\alpha \cos ^{2} \theta_{\Lambda_{c}}\right]\left[1+\alpha_{\Lambda_{c}} \alpha_{\Lambda} \cos \theta_{\Lambda p}\right] } \\
& \times \mathrm{d}\left(\cos \theta_{\Lambda_{c}}\right) \mathrm{d}\left(\cos \theta_{\Lambda_{p}}\right) .
\end{aligned}
$$

The cross-section distribution of Eq. (4.8) applies also to the decay chain, $\Lambda_{c}^{+} \rightarrow \Sigma^{+} \pi^{0}$ and $\Sigma^{+} \rightarrow p \pi^{0}$, with the corresponding identification of indices $b, c$, and $d$.

\section{DIFFERENTIAL DISTRIBUTIONS}

The cross-section distribution (4.8) is a function of two unit vectors $\mathbf{I}_{1}=\mathbf{l}_{\Lambda}$, the direction of motion of the Lambda hyperon in the rest system of the charmed-Lambda hyperon, and $\mathbf{l}_{2}=\mathbf{l}_{p}$ the direction of motion of the proton in the rest system of the Lambda hyperon. In order to handle these vectors we need a common coordinate system, Fig. 1, which we define as follows.

The scattering plane of the reaction $e^{+} e^{-} \rightarrow \Lambda_{c} \bar{\Lambda}_{c}$ is spanned by the unit vectors $\hat{\mathbf{p}}=\mathbf{l}_{\Lambda_{c}}$ and $\hat{\mathbf{k}}=\mathbf{l}_{e^{+}}$, as measured in the c.m. system. We assume the scattering to be to the left, with scattering angle $\theta \geq 0$. If the scattering is to the right we rotate such an event $180^{\circ}$ around the $\mathbf{k}$-axis, so that the scattering appears to be to the left. The

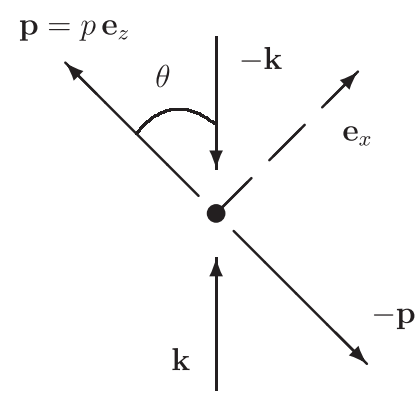

FIG. 1. Momentum vectors in the scattering plane in the c.m. system, with $\mathbf{p}$ the hyperon $\Lambda_{c}$ momentum and $\mathbf{k}$ the positron $e^{+}$ momentum. The $e^{-}$momentum is $-\mathbf{k}$ and the $\bar{\Lambda}_{c}$ momentum $-\mathbf{p}$. Theta $\theta$ is the scattering angle, and $\cos \theta=\hat{\mathbf{p}} \cdot \hat{\mathbf{k}}$. The normal to the scattering plane, $\mathbf{e}_{y}$, points downwards. 
scattering plane makes up the $x z$-plane, with the $y$-axis along the normal to the scattering plane. We choose a righthanded coordinate system with basis vectors

$$
\begin{gathered}
\mathbf{e}_{z}=\hat{\mathbf{p}}, \\
\mathbf{e}_{y}=\frac{1}{\sin \theta}(\hat{\mathbf{p}} \times \hat{\mathbf{k}}), \\
\mathbf{e}_{x}=\frac{1}{\sin \theta}(\hat{\mathbf{p}} \times \hat{\mathbf{k}}) \times \hat{\mathbf{p}} .
\end{gathered}
$$

Expressed in terms of them the initial-state momentum

$$
\hat{\mathbf{k}}=\sin \theta \mathbf{e}_{x}+\cos \theta \mathbf{e}_{z} .
$$

This coordinate system is used for defining the directional angles of the Lambda and the proton. The directional angles of the Lambda hyperon in the charmed-Lambda hyperon rest system are,

$$
\mathbf{l}_{1}=\left(\cos \phi_{1} \sin \theta_{1}, \sin \phi_{1} \sin \theta_{1}, \cos \theta_{1}\right),
$$

whereas the directional angles of the proton in the Lambda hyperon rest system are

$$
\mathbf{l}_{2}=\left(\cos \phi_{2} \sin \theta_{2}, \sin \phi_{2} \sin \theta_{2}, \cos \theta_{2}\right) .
$$

An event of the reaction $e^{+} e^{-} \rightarrow \bar{\Lambda}_{c} \Lambda_{c} ; \Lambda_{c} \rightarrow \Lambda \pi$; $\Lambda \rightarrow p \pi$ is specified by the five dimensional vector $\boldsymbol{\xi}=\left(\theta, \Omega_{1}, \Omega_{2}\right)$, and the differential-cross-section distribution as summarized by Eq. (4.8) reads,

$$
\mathrm{d} \sigma \propto \mathcal{W}(\boldsymbol{\xi}) \mathrm{d} \cos \theta \mathrm{d} \Omega_{1} \mathrm{~d} \Omega_{2} .
$$

At the moment, we are not interested in absolute normalizations. The differential-distribution function $\mathcal{W}(\xi)$ is obtained from Eqs. (1.1), (1.2), (4.4), (4.5), (4.8) and can be expressed as,

$$
\begin{aligned}
\mathcal{W}(\boldsymbol{\xi})= & \mathcal{F}_{0}(\boldsymbol{\xi})+\alpha \mathcal{F}_{1}(\boldsymbol{\xi})+\alpha_{1} \alpha_{2}\left(\mathcal{F}_{2}(\boldsymbol{\xi})+\alpha \mathcal{F}_{3}(\boldsymbol{\xi})\right)+\sqrt{1-\alpha^{2}} \cos (\Delta \Phi)\left(\mathcal{F}_{7}(\boldsymbol{\xi})\right. \\
& \left.+\alpha_{1} \mathcal{F}_{4}(\boldsymbol{\xi})+\beta_{1} \mathcal{F}_{6}(\boldsymbol{\xi})+\gamma_{1}\left(\mathcal{F}_{5}(\boldsymbol{\xi})-\mathcal{F}_{7}(\boldsymbol{\xi})\right)\right)
\end{aligned}
$$

using a set of eight angular functions $\mathcal{F}_{k}(\boldsymbol{\xi})$ defined as:

$$
\begin{aligned}
& \mathcal{F}_{0}(\boldsymbol{\xi})=1, \\
& \mathcal{F}_{1}(\boldsymbol{\xi})=\cos ^{2} \theta, \\
& \mathcal{F}_{2}(\boldsymbol{\xi})=\sin \theta_{1} \sin \theta_{2} \cos \left(\phi_{1}-\phi_{2}\right)+\cos \theta_{1} \cos \theta_{2}, \\
& \mathcal{F}_{3}(\boldsymbol{\xi})=\cos ^{2} \theta \mathcal{F}_{2}(\boldsymbol{\xi}), \\
& \mathcal{F}_{4}(\boldsymbol{\xi})=\sin \theta \cos \theta \sin \theta_{1} \sin \phi_{1}, \\
& \mathcal{F}_{5}(\boldsymbol{\xi})=\sin \theta \cos \theta \sin \theta_{2} \sin \phi_{2}, \\
& \mathcal{F}_{6}(\boldsymbol{\xi})=\sin \theta \cos \theta\left(\cos \theta_{2} \sin \theta_{1} \cos \phi_{1}-\cos \theta_{1} \sin \theta_{2} \cos \phi_{2}\right), \\
& \mathcal{F}_{7}(\boldsymbol{\xi})=\sin \theta \cos \theta \sin \theta_{1} \sin \phi_{1} \mathcal{F}_{2}(\boldsymbol{\xi}) .
\end{aligned}
$$

The differential distribution of Eq. (5.7) involves two parameters related to the $e^{+} e^{-} \rightarrow \Lambda_{c} \bar{\Lambda}_{c}$ reaction that can be determined by data: the ratio of form factors $\alpha$, and the relative phase of form factors $\Delta \Phi$. In addition, the distribution function $\mathcal{W}(\boldsymbol{\xi})$ depends on the weak-decay parameters $\alpha_{1} \beta_{1} \gamma_{1}$ of the charmed-hyperon decay $\Lambda_{c} \rightarrow \Lambda \pi$, and on the weak-decay parameters $\alpha_{2} \beta_{2} \gamma_{2}$ of the hyperon decay $\Lambda \rightarrow p \pi^{-}$. However, the dependency on $\beta_{2}$ and $\gamma_{2}$ drops out. Similarly, integrating over $\mathrm{d} \Omega_{2}$ we get

$$
\begin{aligned}
\mathrm{d} \sigma \propto & {\left[1+\alpha \cos ^{2} \theta\right.} \\
& \left.+\alpha_{1} \sqrt{1-\alpha^{2}} \cos (\Delta \Phi) \sin \theta \cos \theta \sin \theta_{1} \sin \phi_{1}\right] \\
& \times \mathrm{d} \Omega \mathrm{d} \Omega_{1},
\end{aligned}
$$

where now the dependency on $\beta_{1}$ and $\gamma_{1}$ also drops out. The last term in this equation originates with the scalar $\mathbf{P}_{\Lambda_{c}} \cdot \mathbf{N}$. The charmed-hyperon polarization vanishes at $\theta=0^{\circ}, 90^{\circ}$ and $180^{\circ}$.

The distributions presented here will hopefully be of value in the analysis of BESIII data.

\section{MIXED WEAK-ELECTROMAGNETIC HYPERON DECAY}

Now, we extend the formalism to hyperons decaying in two steps, with one being electromagnetic. An example of such a decay chain is $\Lambda_{c}^{+} \rightarrow \Sigma^{0} \pi^{+}$followed by $\Sigma^{0} \rightarrow \Lambda \gamma$. As before we employ indices $b, c$, and $d$ for variables belonging to $\Lambda_{c}^{+}, \Sigma^{0}$, and $\Lambda$. 
The spin-density distribution functions for the weak and electromagnetic transitions are given in Eqs. (4.1) and (3.2),

$$
\begin{gathered}
G_{b}(b, c)=1+\alpha_{b} \mathbf{n}_{b} \cdot \mathbf{l}_{c}+\alpha_{b} \mathbf{n}_{c} \cdot \mathbf{l}_{c}+\mathbf{n}_{b} \cdot \mathbf{L}_{b}\left(\mathbf{n}_{c}, \mathbf{l}_{c}\right), \\
G_{\gamma}(c, d)=1-\mathbf{n}_{c} \cdot \mathbf{l}_{d} \mathbf{l}_{d} \cdot \mathbf{n}_{d} .
\end{gathered}
$$

By folding their product as done for the corresponding product of Eq. (4.3), we get

$$
\begin{aligned}
W_{b}\left(\mathbf{n}_{b} ; \mathbf{l}_{c}, \mathbf{l}_{d}\right) & =\left\langle G_{b}(b, c) G_{\gamma}(c, d)\right\rangle_{c d} \\
& =U_{b}+\mathbf{n}_{b} \cdot \mathbf{V}_{b},
\end{aligned}
$$

with

$$
U_{b}=1, \quad \mathbf{V}_{b}=\alpha_{b} \mathbf{l}_{c} .
$$

These expressions for $U_{b}$ and $\mathbf{V}_{b}$ are noteworthy. They are in fact the same as those of a one-step $b \rightarrow c \pi$ decay, Eq. (2.5). Hence, the electromagnetic decay does not add any structure, Eq. (6.4) is independent of $\mathbf{1}_{d}$.

The initial state spin distribution function for hyperon $b$ produced in $e^{+} e^{-}$annihilation is as above, Eq. (4.6),

$$
S_{b}\left(\mathbf{P}_{b}\right)=1+\mathbf{P}_{b} \cdot \mathbf{n}_{b}
$$

Folding this distribution function with the decay distribution function of Eq. (6.3), we obtain

$$
\begin{aligned}
W_{b}\left(\mathbf{P}_{b} ; \mathbf{l}_{c}, \mathbf{l}_{d}\right) & =\left\langle S_{b}\left(\mathbf{P}_{b}\right) G_{b}(b, c) G_{\gamma}(c, d)\right\rangle_{b c d} \\
& =U_{b}+\mathbf{P}_{b} \cdot \mathbf{V}_{b} .
\end{aligned}
$$

As noted earlier this is equivalent to making the replacement $\mathbf{n}_{b} \rightarrow \mathbf{P}_{b}$ in Eq. (6.3). We also notice if we manage to determine $U_{b}$ and $\mathbf{V}_{b}$ of Eq. (6.6), the only parameter that can be fixed is $\alpha_{b}$, a meager return.

The expression for the cross-section distribution for $\Lambda_{c}^{+}$production and subsequent decays $\Lambda_{c}^{+} \rightarrow \Sigma^{0} \pi^{+}$and $\Sigma^{0} \rightarrow \Lambda \gamma$ is

$$
\mathrm{d} \sigma \propto\left[\mathcal{R} U_{\Lambda_{c}}+\mathcal{S} \mathbf{N} \cdot \mathbf{V}_{\Lambda_{c}}\right] \mathrm{d} \Omega_{\Lambda_{c}} \mathrm{~d} \Omega_{\Sigma} \mathrm{d} \Omega_{\Lambda},
$$

$$
R^{-1}(\alpha, \beta, \gamma)=\left(\begin{array}{lll}
\cos \alpha \cos \beta \cos \gamma-\sin \alpha \sin \gamma & \cos \gamma \cos \beta \sin \alpha+\sin \gamma \cos \alpha & -\sin \beta \cos \gamma \\
-\sin \gamma \cos \beta \cos \alpha-\cos \gamma \sin \alpha & -\sin \gamma \cos \beta \sin \alpha+\cos \gamma \cos \alpha & \sin \beta \sin \gamma \\
\cos \alpha \sin \beta & \sin \gamma \sin \beta & \cos \beta
\end{array}\right),
$$

with $\bar{r}_{s}=R^{-1}(\alpha, \beta, \gamma) \bar{r}_{b}$ and $\alpha \beta \gamma$ the Euler angles.

The angular integrations can be expressed in terms of the Euler angles, as

$$
\mathrm{d} \Omega_{c} \mathrm{~d} \Omega_{d}=\mathrm{d}\left(\cos \theta_{c d}\right) \mathrm{d} \alpha \mathrm{d}(\cos \beta) \mathrm{d} \gamma .
$$


The expression to be integrated, Eq. (4.5), reads

$$
\begin{aligned}
U_{b}+\mathbf{P} \cdot \mathbf{V}_{b}= & 1+\alpha_{b} \alpha_{c} \mathbf{l}_{c} \cdot \mathbf{l}_{d}+\alpha_{b} \mathbf{P} \cdot \mathbf{l}_{c}+\gamma_{b} \mathbf{P} \cdot \mathbf{l}_{d} \\
& +\left(1-\gamma_{b}\right) \mathbf{P} \cdot \mathbf{l}_{c} \mathbf{l}_{d} \cdot \mathbf{l}_{c}+\beta_{b} \mathbf{P} \cdot\left(\mathbf{l}_{d} \times \mathbf{l}_{c}\right),
\end{aligned}
$$

with $\mathbf{P}$ along the $Z$-direction.

Now, we note that terms proportional to $\mathbf{P} \cdot \mathbf{l}_{c}$ or $\mathbf{P} \cdot \mathbf{l}_{d}$ vanish upon integration over angles $\alpha$ or $\gamma$. Therefore,

$$
\begin{aligned}
\int \mathrm{d} \Omega_{c} \mathrm{~d} \Omega_{d}\left(U_{b}+\mathbf{P} \cdot \mathbf{V}_{b}\right) \\
=4 \pi^{2} \int \mathrm{d}\left(\cos \theta_{c d}\right) \mathrm{d}(\cos \beta)\left(1+\alpha_{b} \alpha_{c} \cos \theta_{c d}\right. \\
\left.\quad-\beta_{b} \alpha_{c} P \sin \theta_{c d} \cos \beta\right) \\
=8 \pi^{2} \int \mathrm{d}\left(\cos \theta_{c d}\right)\left(1+\alpha_{b} \alpha_{c} \cos \theta_{c d}\right) .
\end{aligned}
$$

This result leads to Eq. (4.11).
[1] G. Fäldt, Eur. Phys. J. A 52, 141 (2016).

[2] G. Fäldt and A. Kupsc, Phys. Lett. B 772, 16 (2017).

[3] J. D. Bjorken and S. D. Drell, Relativistic Quantum Mechanics (McGraw-Hill, New York, 1964).
[4] L. B. Okun, Leptons and Quarks (North-Holland, Amsterdam, 1982).

[5] C. Materniak, Nucl. Phys. B, Proc. Suppl. 187, 208 (2009).

[6] D. Wang, R.-G. Ping, L. Li, X.-R. Lyu, and Y.-H. Zheng, Chin. Phys. C 41, 023106 (2017). 